\title{
Predictability of antitumor efficacy of cetuximab plus irinotecan based on skin rash severity according to observation period in patients with metastatic colorectal cancer following failure of fluorouracil, irinotecan and oxaliplatin
}

\author{
YOSHIKI HORIE ${ }^{1,2}$, KENTARO YAMAZAKI ${ }^{2}$, TARO FUNAKOSHI ${ }^{2}$, SATOSHI HAMAUCHI $^{2}$, \\ HIROYA TANIGUCHI $^{2}$, TAKAHIRO TSUSHIMA ${ }^{2}$, AKIKO TODAKA $^{2}$, NOZOMU MACHIDA $^{2}$, \\ KEISEI TAKU $^{2}$, AKIRA FUKUTOMI ${ }^{2}$, YUSUKE ONOZAWA ${ }^{2}$, HIROFUMI YASUI ${ }^{2}$, TAKURO MIZUKAMI ${ }^{1}$, \\ NAOKI IZAWA ${ }^{1}$, MAMI HIRAKAWA ${ }^{1}$, TAKASHI TSUDA ${ }^{1}$, TAKAKO NAKAJIMA ${ }^{1}$ and NARIKAZU BOKU ${ }^{1}$ \\ ${ }^{1}$ Department of Clinical Oncology, St. Marianna University School of Medicine, Kawasaki, Kanagawa 216-8511; \\ ${ }^{2}$ Division of Gastrointestinal Oncology, Shizuoka Cancer Center, Shizuoka, Shizuoka 411-8777, Japan
}

Received November 16, 2014; Accepted March 30, 2015

DOI: $10.3892 /$ mco.2015.586

\begin{abstract}
The efficacy of cetuximab correlates with the severity of skin toxicity, although its onset may vary. The aim of this retrospective study was to investigate the optimal observation period for skin rash as a predictor of the efficacy of cetuximab plus irinotecan. The subjects comprised 33 patients with KRAS wild-type metastatic colorectal cancer (mCRC) who had received prior chemotherapy with fluorouracil, irinotecan and oxaliplatin. The response rate (RR), progression-free survival (PFS) and overall survival (OS) were compared according to the presence or absence of $\geq$ grade 2 skin rash within $2,4,6$, or 8 weeks following cetuximab initiation. The overall RR was $45 \%$ (15/33) and the median PFS and OS were 188 and 383 days, respectively. A total of 26 patients experienced $\geq$ grade 2 skin rash within 8 weeks. The proportion of responders among patients who developed $\geq$ grade 2 skin rash (severe group) decreased depending on the duration of the observation period (50\% within 8 weeks), whereas the proportion of non-responders among patients with <grade 2 skin rash (mild group) increased (71\% within 8 weeks). Similarly, the proportion of patients with an unfavorable prognosis (PFS $<6$ months, OS $<1$ year) in the mild group increased $(86 \%$ for PFS and $71 \%$ for OS within 8 weeks), whereas the proportion of those with a favorable prognosis in the severe group remained stable (73\% for PFS and $62 \%$ for OS within 8 weeks). Therefore, the absence of $\geq$ grade 2 skin rash within 8 weeks may be predictive of unfavorable efficacy of cetuximab plus irinotecan in mCRC patients.
\end{abstract}

Correspondence to: Dr Yoshiki Horie, Department of Clinical Oncology, St. Marianna University School of Medicine, 2-16-1 Sugao, Miyamae-ku, Kawasaki, Kanagawa 216-8511, Japan E-mail: holt802@ybb.ne.jp

Key words: colorectal cancer, cetuximab, skin rash, predictability

\section{Introduction}

Metastatic colorectal cancer (mCRC) treatment has advanced over the last decade. Chemotherapeutic treatment generally includes 3 active cytotoxic agents, namely fluorouracil (FU), irinotecan and oxaliplatin, irrespective of the administration sequence (1), whereas biological therapies have further improved each treatment regimen.

Cetuximab, a chimeric monoclonal immunoglobulin that binds to the epidermal growth factor receptor (EGFR), blocks signal transduction, modulates tumor growth and mediates antibody-dependent cell-mediated cytotoxicity. A number of trials of cetuximab as monotherapy and as part of combination therapy for $\mathrm{mCRC}$ have been conducted. Initially, cetuximab combined with irinotecan yielded a higher response rate compared with cetuximab monotherapy for irinotecan-refractory mCRC patients, suggesting that cetuximab may restore irinotecan chemosensitivity (2). Additionally, cetuximab has been proven to be effective as a single agent, with objective response rates of $9-12 \%$, and has been associated with a survival benefit over best supportive care (3). As regards first-line treatment, trials in which cetuximab was added to infusional FU-based chemotherapy combined with irinotecan (4) or oxaliplatin (5) demonstrated improvements in the clinical outcomes of KRAS wild-type mCRC patients. The KRAS gene status is currently an important predictive marker of cetuximab efficacy.

An acne-like or maculopapular rash, a characteristic side effect of EGFR blockade, is considered to be caused by disturbing the role of EGFR in maintaining skin integrity. A number of clinical trials have reported that the grade of the most severe skin rash observed throughout the entire treatment course is strongly correlated with cetuximab efficacy. Therefore, skin toxicity is considered to be another marker of cetuximab efficacy. The ability to predict cetuximab efficacy from skin toxicity severity as soon as possible after treatment initiation would be very useful. However, the onset of severe 
skin toxicity varies among patients and the precise time point at which the efficacy of cetuximab may be predicted by the severity of skin toxicity has not been clearly determined. The aim of this retrospective study was to investigate the association between the presence or absence of a severe skin rash within 2, 4, 6, or 8 weeks following initiation of cetuximab plus irinotecan chemotherapy and the efficacy of this combination treatment for $\mathrm{mCRC}$ patients following failure of $\mathrm{FU}$, irinotecan and oxaliplatin.

\section{Materials and methods}

Patients. A search through the Division of Gastrointestinal Oncology database at the Shizuoka Cancer Center (Shizuoka, Japan) identified $60 \mathrm{mCRC}$ patients who were treated with cetuximab-containing regimens, initiated between September, 2008 and December, 2009. The selection criteria for this retrospective study were as follows: refractoriness to treatment with FU, irinotecan and oxaliplatin; confirmed KRAS codon 12 and 13 (exon 2) wild-type status; performance status $<2$; treatment with cetuximab plus irinotecan; no prior anti-EGFR drug treatment history; adequate organ function; no severe medical conditions; and follow-up $>2$ months, regardless of the chemotherapy duration. Following exclusion of 27 patients (not proven KRAS wild-type status, $n=10$; cetuximab monotherapy, $n=8$; not refractory to all 3 drugs, $\mathrm{n}=4$; poor performance status, $\mathrm{n}=1$; prior anti-EGFR drug treatment, $n=1$; jaundice, $n=1$; severe infection, $n=1$; short follow-up, $n=1$ ), 33 patients met all the selection criteria and were included in the analysis. Data regarding prior treatments, baseline patient characteristics, treatment duration, adverse events, antitumor effects (response, progression-free survival and overall survival) and the skin rash appearance date and severity were collected by reviewing the electronic medical charts.

This study was approved by the Shizuoka Cancer Center Institutional Review Board.

Treatment. The patients were treated with a combination of cetuximab plus irinotecan (weekly cetuximab administration of $250 \mathrm{mg} / \mathrm{m}^{2}$ after a $400-\mathrm{mg} / \mathrm{m}^{2}$ initial loading dose and bi-weekly irinotecan administration of $150 \mathrm{mg} / \mathrm{m}^{2}$ ). Cetuximab administration was skipped when grade 3 or 4 skin toxicities were observed and the dose was reduced by $20 \%$ upon resumption. Irinotecan skipping and dose reduction were performed at the physician's discretion, according to the severity of adverse events. Preemptive skin care, including application of skin moisturizer twice a day in the morning and evening after bathing and application of sunscreen (SPF $>15$, UVA and UVB protection) to exposed skin areas prior to sun exposure, was administered with the first dose of cetuximab and continued throughout the therapy; no prophylactic antibiotics were administered.

Evaluation. All adverse events, including skin toxicities, were weekly evaluated and graded according to the Common Terminology Criteria for Adverse Events, version 3.0 (http:// ctep.cancer.gov/protocolDevelopment/electronic_applications/ ctc.htm). The patients were divided into two groups according to the presence or absence of $\geq$ grade 2 skin rash during each observation period (2, 4, 6 and 8 weeks) following cetuximab initiation. All the patients were included in all the skin rash evaluations (e.g., any patient whose treatment was discontinued prior to 8 weeks after initiation was included in skin toxicity evaluations at each obserbation period until 8 weeks), regardless of the skin rash severity. Response to treatment was evaluated by computed tomography, which was repeated approximately every 2 months, according to the Response Evaluation Criteria in Solid Tumors, version 1.0 (http://jnci. oxfordjournals.org/content/92/3/205.full). Progression-free survival was calculated from the date of cetuximab initiation until the date of disease progression, as determined clinically or radiologically, or the date of death, and was censored at the date of treatment discontinuation without disease progression. Similarly, overall survival was calculated from the date of cetuximab initiation to the date of death from any cause and was censored at the last survival date.

Statistical analysis. Progression-free survival and overall survival curves were generated using the Kaplan-Meier method and compared using the log-rank test between the two groups of patients divided according to the presence or absence of $\geq$ grade 2 skin rash for each observation period (2, 4, 6 and 8 weeks and the entire treatment course). Patients who achieved complete or partial response were defined as responders and the remaining as non-responders. Progression-free and overall survival were stratified by the median value, to represent favorable or unfavorable prognosis. Correlations between the presence or absence of $\geq$ grade 2 skin rash within each observation period and treatment efficacy in terms of response (favorable, responder; unfavorable, non-responder), progression-free survival (favorable, $>6$ months; unfavorable, $<6$ months) and overall survival (favorable, $>1$ year; unfavorable, $<1$ year) were investigated and the proportion of patients with favorable efficacy among those with $\geq$ grade 2 skin rash and of that of patients with unfavorable efficacy among those with <grade 2 skin rash were calculated for each observation period. All the analyses were performed using JMP software, version 8.2 (SAS Institute, Cary, NC, USA).

\section{Results}

Patient background. The patient background characteristics are summarized in Table I. The median patient age was 60 years (range, 27-76 years) and 91\% of the patients had a performance status of 0 or 1 . All the patients had at least 1 measurable lesion for response evaluation and 28 patients $(85 \%)$ had $\leq 2$ metastatic lesions. Prior treatment had been discontinued due to disease progression in all the patients.

Toxicities. The median number of cetuximab administrations was 22 (range, 3-104). Cetuximab administration was skipped a total of 50 times in 21 patients and fatigue was the most frequent reason for skipping treatment $(n=9)$. Cetuximab was skipped 3 times because of skin rash. The cetuximab doses were reduced once in 2 patients due to fatigue and rash. Treatment was discontinued due to disease progression in 31 patients and due to adverse events in 2 patients (pneumonitis after 6 cycles, $\mathrm{n}=1$; and fatigue after 7 cycles, $\mathrm{n}=1$ ). 
Table I. Patient background characteristics.

\begin{tabular}{lc}
\hline Characteristics & $\begin{array}{c}\text { No. of patients }(\%) \\
(\mathrm{n}=33)\end{array}$ \\
\hline $\begin{array}{l}\text { Median age, years (range) } \\
\text { Gender }\end{array}$ & $60(27-76)$ \\
Male/female & $18 / 15(45 / 55)$ \\
Performance status & $13 / 17 / 3(39 / 52 / 9)$ \\
0/1/2 & $31 / 2(94 / 6)$ \\
Primary sites & \\
Colon/rectum & $10 / 18 / 5(30 / 55 / 15)$ \\
No. of metastatic organs & \\
1/2/3 & $27 / 5 / 1(82 / 15 / 3)$ \\
No. of prior chemotherapy cycles \\
2/3/4
\end{tabular}

The worst grades of the major adverse events observed during the entire treatment course are shown in Table II. Skin rash was the most common non-hematological adverse event and it was observed in all the patients (grade $1, n=5$; grade $2, n=24$; and grade $3, n=4$ ). The number of patients who experienced skin rash increased along with the treatment course duration (Fig. 1). The median time to the first appearance of $\geq$ grade 1 skin rash was 10 days (range, 6-48 days) and that to the first appearance of $\geq$ grade 2 skin rash 21 days (range, 7-116 days). A total of 11, 14, 17 and 26 patients developed $\geq$ grade 2 skin rash within 2, 4, 6 and 8 weeks following cetuximab initiation, respectively.

Efficacy. Although there were no complete responses, 15 patients $(45 \%)$ achieved a partial response and 12 patients
Table II. Worst adverse event grade throughout the entire course of treatment ${ }^{\mathrm{a}}$.

\begin{tabular}{llll}
\hline & \multicolumn{3}{c}{ Grade } \\
\cline { 2 - 4 } Adverse events & 3 & 4 & $3 / 4(\%)$ \\
\hline Neutropenia & 3 & 2 & $5(15.2)$ \\
Acne-like skin rash & 4 & - & $4(12.1)$ \\
Paronychia & 4 & - & $4(12.1)$ \\
Loss of appetite & 3 & 0 & $3(9.0)$ \\
Febrile neutropenia & 0 & 1 & $1(3.0)$ \\
Interstitial pneumonia & 0 & 1 & $1(3.0)$ \\
Diarrhea & 1 & 0 & $1(3.0)$ \\
Vomiting & 1 & 0 & $1(3.0)$ \\
Infusion reaction & 0 & 0 & $0(0.0)$ \\
Stomatitis & 0 & - & $0(0.0)$ \\
Cutaneous dryness & 0 & - & $0(0.0)$ \\
Fatigue & 0 & 0 & $0(0.0)$ \\
\hline
\end{tabular}

${ }^{\mathrm{a} C e t u x i m a b}$ plus irinotecan.

(36\%) maintained stable disease. With a median follow-up period of 1,209 days (range, 1,131-1,286 days) for survivors, the median progression-free survival was 188 days and the median survival 383 days (Fig. 2). The median time-to-response among the responders was 8.7 weeks (range, 4-35 weeks).

Progression-free survival and overall survival according to the presence or absence of $\geq$ grade 2 skin rash. The progression-free and overall survival curves of the patients who did and those who did not experience $\geq$ grade 2 skin rash within 2, 4, 6 and 8 weeks and over the entire treatment course after cetuximab initiation are preseented in Fig. 3.

The median progression-free survival according to skin rash severity (<grade 2, mild; and $\geq$ grade 2 , severe) throughout the entire treatment course was 58 days for the mild group $(n=6)$ and 210 days for the severe group $(n=27$; hazard ratio $[\mathrm{HR}]=0.241 ; 95 \%$ confidence interval $[\mathrm{CI}]: 0.097-0.680$;

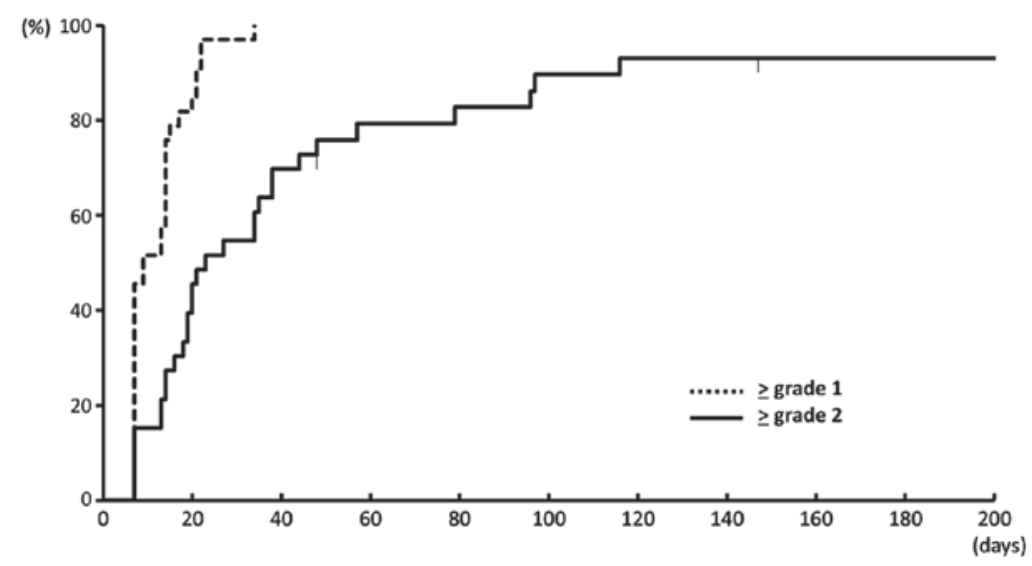

Figure 1. Onset of skin rash following initiation of cetuximab plus irinotecan combination chemotherapy. The number of patients who developed a skin rash increased along with the treatment course duration. 


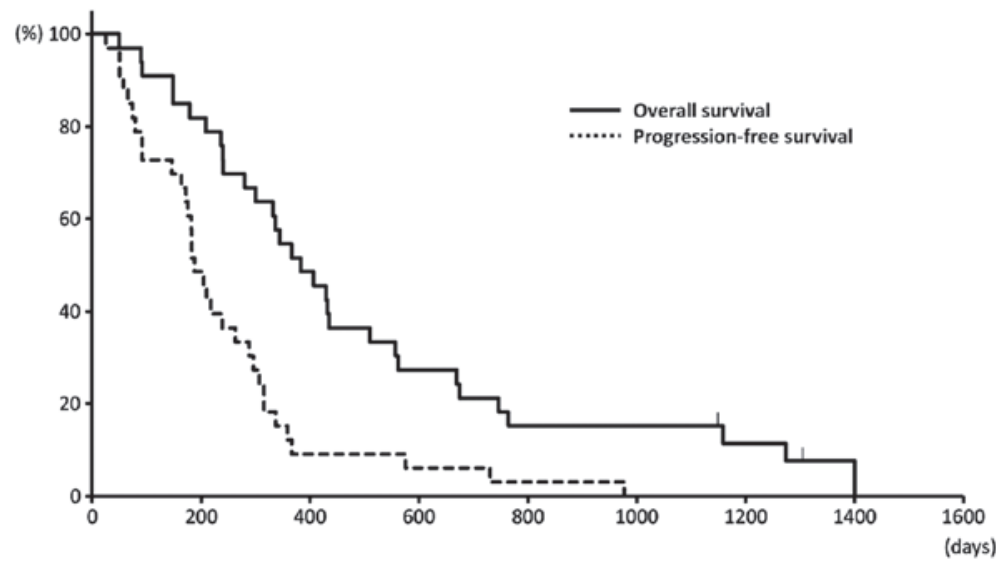

Figure 2. Progression-free and overall survival of the patients. With a median follow-up period of 1,209 days (range, 1,131-1,286 days) for survivors, the median progression-free survival was 188 days and the median overall survival was 383 days.
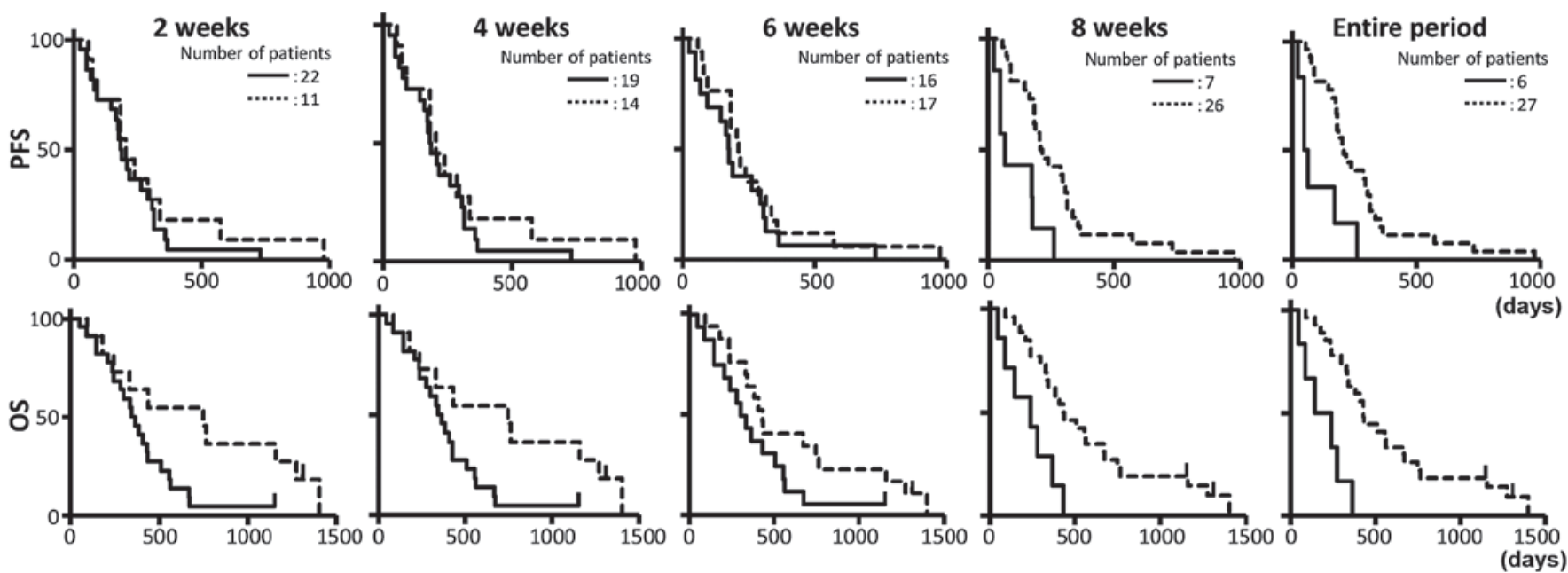

Figure 3. Progression-free and overall survival of patients with or without grade $\geq 2$ skin rash within each observation period. The progression-free survival (PFS) and overall survival (OS) curves of the patients who did and did not experience grade $\geq 2$ skin rash within 2, 4,6 and 8 weeks and over the entire treatment course following cetuximab initiation. Dashed line, patients with severe skin rash; and solid line, patients without severe skin rash.

$\mathrm{P}=0.0015)$ and the median overall survival was 195 and 432 days, respectively $(\mathrm{HR}=0.181 ; 95 \% \mathrm{CI}: 0.065-0.544$; $\mathrm{P}=0.0003)$. The overall response rate was $16.7 \%(1 / 6)$ and $51.9 \%(14 / 27)$ in the mild and severe groups, respectively.

The median progression-free survival was 205 days in the 11 patients who developed $\geq$ grade 2 skin rash within 2 weeks after cetuximab initiation and 188 days in the 22 patients who did not $(\mathrm{HR}=0.740$; 95\% CI: 0.333-1.534; $\mathrm{P}=0.4294)$. Similarly, the median progression-free survival in the mild and severe skin rash groups within 4,6 and 8 weeks were 183 and 208 days $(n=19 / 14 ;$ HR=0.793; 95\% CI: 0.381-1.597; $\mathrm{P}=0.5159), 175$ and 210 days $(\mathrm{n}=16 / 17 ; \mathrm{HR}=0.743$; 95\% CI: $0.368-1.501 ; \mathrm{P}=0.3972)$ and 66 and 210 days $(\mathrm{n}=7 / 26 ; \mathrm{HR}=0.247 ; 95 \%$ CI: 0.102-0.658; $\mathrm{P}=0.0012)$, respectively. The median overall survival in the mild and severe groups within 2, 4, 6 and 8 weeks was 355 and 747 days (HR=0.359; 95\% CI: 0.134-0.845; $\mathrm{P}=0.0220), 366$ and 421 days $(\mathrm{HR}=0.504 ; 95 \% \mathrm{CI}: 0.217-1.101 ; \mathrm{P}=0.0887), 319$ and 430 days $(\mathrm{HR}=0.525 ; 95 \% \mathrm{CI}: 0.242-1.121 ; \mathrm{P}=0.0901)$ and 241 and 433 days $(\mathrm{HR}=0.263$; 95\% CI: 0.106-0.709; $\mathrm{P}=0.0026)$, respectively.
Favorable or unfavorable efficacy and the presence or absence of $\geq$ grade 2 skin rash. The proportions of patients with favorable efficacy in the severe group and of patients with unfavorable efficacy in the mild group within each observation period $(2,4,6$ and 8 weeks and the entire treatment course) in terms of response, progression-free survival and overall survival are presented in Fig. 4. The proportion of responders in the severe group decreased during the observation period $(64 \%$ within 2 weeks, $64 \%$ within 4 weeks, $53 \%$ within 6 weeks, $50 \%$ within 8 weeks and 52\% during the entire treatment couse) and that of non-responders in the mild group increased $(64,68,63,71$ and $83 \%$, respectively) (Fig. 4A). Regarding progression-free survival (Fig. 4B), the proportion of patients with a favorable progression-free survival ( $\geq 6$ months) in the severe group exhibited no significant changes during the observation period $(73,71,76,73$ and $70 \%$, respectively), whereas that of patients with unfavorable progression-free survival ( $<6$ months) increased in the mild group $(45,47,56,86$ and $83 \%$, respectively). Similarly, the proportions of patients with favorable survival ( $\geq 1$ year) in the severe group exhibited no significant changes during the 

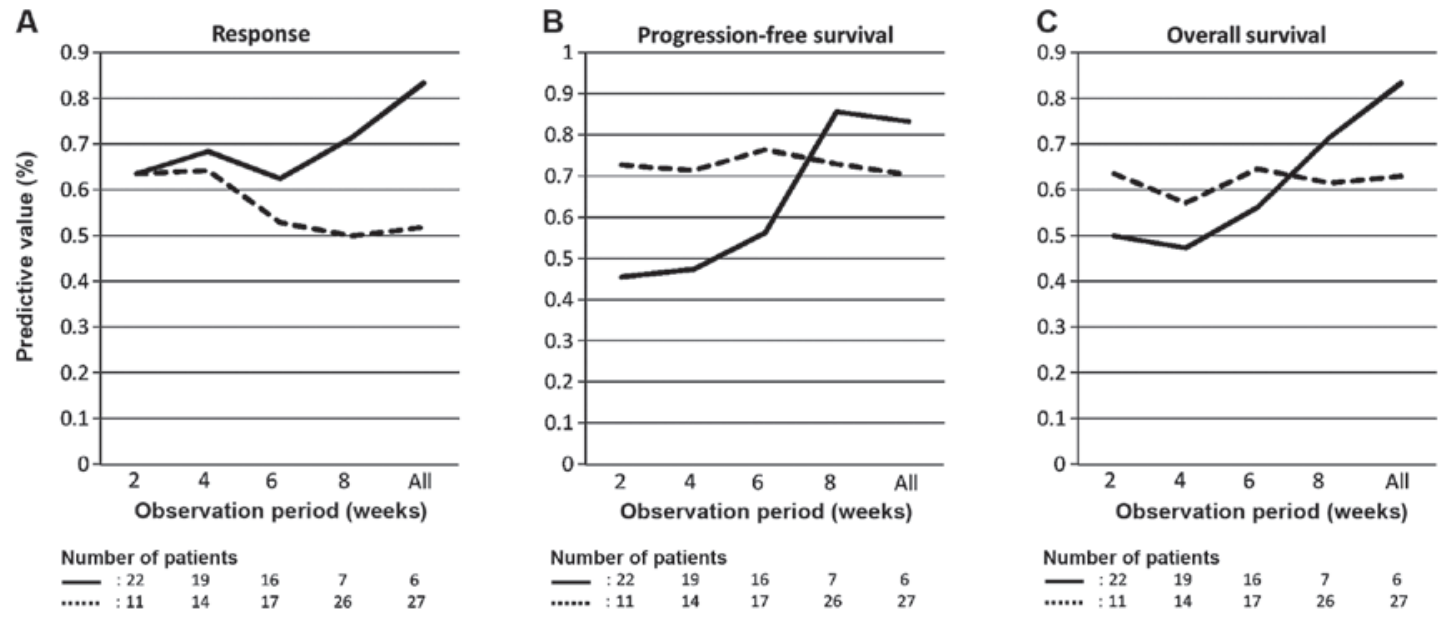

Figure 4. Prediction of favorable or unfavorable efficacy in patients with or without grade $\geq 2$ skin rash within each observation period. The proportions of patients with favorable efficacy in the severe rash (grade $\geq 2$ ) group (positive predictive value, dashed line) and of patients with unfavorable efficacy in the mild rash (grade <2) group (negative predictive value, solid line) within each observation period $(2,4,6$, and 8 weeks and the entire treatment course) in terms of (A) response, (B) progression-free survival (PFS) and (C) overall survival (OS). Favorable efficacy is defined as complete or partial response, PFS $>6$ months and OS $>12$ months. Unfavorable efficacy is defined as stable or progressive disease, PFS $<6$ months and OS $<12$ months. All, entire treatment course.

observation period $(64,57,65,62$ and $63 \%$, respectively), whereas that of patients with unfavorable survival $(<1$ year $)$ increased in the mild group $(50,47,56,71$ and $83 \%$, respectively) (Fig. 4C).

\section{Discussion}

Immediately following approval of cetuximab in Japan in July, 2008, cetuximab plus irinotecan became one of the most commonly used chemotherapeutic regimens (6) for patients with failure of prior chemotherapy with 3 active drugs, as this combination therapy has been reported to be superior to cetuximab monotherapy following irinotecan failure (2). For this reason, the subjects of the present study were limited to those treated with cetuximab plus irinotecan for mCRC following failure of prior chemotherapy with fluorouracil, irinotecan and oxaliplatin. As cetuximab was found to be ineffective for patients with KRAS mutations, investigations of the efficacy of cetuximab-containing chemotherapies should be based on patients with KRAS wild-type cancers. However, as the KRAS status test had not been approved in Japan until April, 2010, cetuximab was initially used regardless of the KRAS status. Therefore, 10 patients without confirmed wild-type KRAS status were excluded from this study.

Cetuximab-induced skin toxicities, such as acne-like skin rashes and dry skin, are a well-known occurrence. These toxicities are considered to represent the pharmacodynamics of EGFR signaling inhibitors in the skin. As internalization strengthens EGFR signaling inhibition, antibody-based drugs (e.g., cetuximab and panitumumab) are associated with a higher incidence of severe skin toxicities compared with small-molecule tyrosine kinase inhibitors, such as gefitinib (7). The most common sites for the appearance of skin toxicities are the head, face, neck, shoulders, chest and back. In previous reports, such as the BOND (2), EPIC (8), OPUS (5) and CRYSTAL trials (4), the worst skin rash severity observed during the entire treatment course correlated strongly with cetuximab efficacy. While efficacy parameters, such as response, progression-free survival and overall survival in this study appeared to be superior to those reported by earlier trials using the same chemotherapy regimen after failure of the 3 cytotoxic drugs, this study may contain some selection bias caused by discriminating patients for whom irinotecan-containing combinations were not considered feasible. Even with these favorable clinical outcomes, the correlation between skin rash severity during the treatment course and cetuximab plus irinotecan efficacy was recapitulated in this study.

In clinical practice, the first radiological imaging response evaluation is usually performed at approximately 8 weeks and it would be useful if anti-EGFR antibody efficacy was predicted as favorable or unfavorable based on skin rash severity prior to the first response evaluation. However, the proportion of responders among patients with $\geq$ grade 2 skin rash remained at 50-60\% and marginally decreased throughout the observation period, whereas the overall response rate was as high as $45 \%$. Thus, it may be difficult to predict responses from skin rash severity prior to the first response evaluation. Moreover, the progression-free and overall survival HRs in the two groups (mild and severe) were higher until 6 weeks compared with those obtained at 8 weeks and during the entire treatment course. These results suggest that the differences in progression-free and overall survival between patients with and those without $\geq$ grade 2 skin rash were increasing along with the treatment course, with the skin rash severity at 8 weeks suggested to be a better prognostic marker compared with that within shorter observation periods. In the EVEREST trial, the cetuximab dose was increased in patients who did not develop severe skin toxicities until 3 weeks. Although the incidence of $\geq$ grade 2 skin reactions increased by $21 \%$ in the increased-dose group compared with that in the standard-dose group, there was no difference in survival (9). Together with the results of this study, a 3-week observation period for deciding dose increase based on skin rash severity may be insufficient to predict cetuximab efficacy in terms of overall survival.

Using receiver-operating characteristic curves, the optimal observation periods for the presence or absence of $\geq$ grade 2 
skin rash as a predictor of favorable or unfavorable efficacy were inconsistent for all efficacy endpoints; the optimal observation periods were 4 weeks for response, 8 weeks for progression-free survival and the entire treatment course for overall survival (data not shown). Regarding sensitivity and specificity, there were no significant differences in differentiation ability among the 4 observation periods (2, 4, 6 and 8 weeks) for each efficacy endpoint. Therefore, it may be difficult to determine the optimal observation period for the presence or absence of $\geq$ grade 2 skin rash in order to differentiate patients exhibiting favorable or unfavorable efficacy of cetuximab plus irinotecan.

However, from a clinical point of view, prediction should be based on the probability of favorable or unfavorable events for each patient. In this study, the proportion of non-responders among those without $\geq$ grade 2 skin rash increased along with the treatment course, whereas only 1 patient without $\geq$ grade 2 skin rash achieved a partial response. Skin rash severity is considered to be determined by each patient's genomic background; skin and tumors share a common genetic background, including genetic polymorphisms. It may be considered that a genetic background that causes a severe skin rash is required to achieve satisfactory cetuximab-mediated tumor shrinkage in the majority of the patients. Indeed, the negative predictive value of unfavorable efficacy without $\geq$ grade 2 skin rash within 8 weeks was $>80 \%$ in terms of response, progression-free survival and overall survival, whereas only 5 patients in this study developed $\geq$ grade 2 skin rash after 8 weeks. Therefore, the absence of a $\geq$ grade 2 skin rash within 8 weeks may be predictive of an unfavorable clinical outcome, although the number of patients without a severe skin rash decreased along with the treatment course. Certain trials were recently conducted on the prevention of skin toxicities. The STEPP trial demonstrated that anti-EGFR antibody-induced skin toxicities may be prevented to some degree by the prophylactic use of humectants, sunscreens, topical steroids and oral antibiotics for 6 weeks (10). With the introduction of prophylactic treatments for skin toxicity in clinical practice, the incidence of severe skin toxicity has decreased. Therefore, it may be difficult to predict unfavorable cetuximab efficacy from the absence of severe skin toxicity if prophylactic treatments have been used.

Regarding positive prediction, the proportion of patients who obtained favorable efficacy among those with severe skin rash remained stable throughout the entire observation period. These results suggest that there may be an underlying mechanism of cetuximab resistance besides KRAS in some populations. In addition to the KRAS mutation in exon 2, for which there is a commonly available clinical test, KRAS mutations in exons 3 and 4, NRAS and BRAF may be associated with cetuximab resistance. However, the tumors of some patients do not respond to cetuximab, despite negative results for all the aforementioned gene mutations and the presence of severe skin toxicity. Therefore, the development of a novel biomarker to predict favorable cetuximab efficacy is required.

In conclusion, although it is difficult to predict favorable efficacy of cetuximab plus irinotecan from skin rash severity within 8 weeks, the absence of a severe skin rash within 8 weeks may be predictive of unfavorable progression-free and overall survival.

\section{References}

1. Grothey A, Sargent D, Goldberg RM and Schmoll HJ: Survival of patients with advanced colorectal cancer improves with the availability of fluorouracil-leucovorin, irinotecan and oxaliplatin in the course of treatment. J Clin Oncol 22: 1209-1214, 2004.

2. Cunningham D, Humblet Y, Siena S, et al: Cetuximab monotherapy and cetuximab plus irinotecan in irinotecan-refractory metastatic colorectal cancer. New Engl J Med 351: 337-345, 2004.

3. Jonker DJ, O'Callaghan CJ, Karapetis CS, et al: Cetuximab for the treatment of colorectal cancer. N Engl J Med 357: 2040-2048, 2007.

4. Van Cutsem E, Köhne CH, Hitre E, et al: Cetuximab and chemotherapy as initial treatment for metastatic colorectal cancer. N Engl J Med 360: 1408-1417, 2009.

5. Bokemeyer C, Bondarenko I, Makhson A, et al: Fluorouracil, leucovorin and oxaliplatin with and without cetuximab in the first-line treatment of metastatic colorectal cancer. J Clin Oncol 27: 663-671, 2009.

6. Ishiguro M, Watanabe $\mathrm{T}$, Yamaguchi $\mathrm{K}$, et al: A Japanese post-marketing surveillance of cetuximab (Erbitux $\left.{ }^{\circledR}\right)$ in patients with metastatic colorectal cancer. Jpn J Clin Oncol 42: 287-294, 2012.

7. Mendelsohn J and Baselga J: Status of epidermal growth factor receptor antagonists in the biology and treatment of cancer. J Clin Oncol 21: 2787-2799, 2003.

8. Sobrero AF, Maurel J, Fehrenbacher L, et al: EPIC: phase III trial of cetuximab plus irinotecan after fluoropyrimidine and oxaliplatin failure in patients with metastatic colorectal cancer. J Clin Oncol 26: 2311-2319, 2008.

9. Van Cutsem E, Humblet Y, Gelderblom H, et al: Cetuximab dose-escalation study in patients with metastatic colorectal cancer (mCRC) with no or slight skin reactions on cetuximab standard dose treatment (EVEREST): Pharmacokinetic and efficacy data of a randomized study. JOP 10: 361-365, 2007.

10. Lacouture ME, Mitchell EP, Piperdi B, et al: Skin toxicity evaluation protocol with panitumumab (STEPP), a phase II, open-label, randomized trial evaluating the impact of a pre-emptive skin treatment regimen on skin toxicities and quality of life in patients with metastatic colorectal cancer. J Clin Oncol 28: 1351-1357, 2010. 